Research Article

\title{
Effect of Basketball on Improving the Health of Obese People under the Monitoring of Internet of Things Technology
}

\author{
Zhaoxin Liu $^{1,2}$ and Junchang Kan $\mathbb{D}^{1}$ \\ ${ }^{1}$ Harbin Sport University, Harbin 150008, Heilongjiang, China \\ ${ }^{2}$ Physical Education and Sports Training College, Harbin Sport University, Harbin 150008, Heilongjiang, China \\ Correspondence should be addressed to Junchang Kan; kan1970hsu@163.com
}

Received 19 May 2021; Revised 13 June 2021; Accepted 13 July 2021; Published 31 July 2021

Academic Editor: Sang-Bing Tsai

Copyright (c) 2021 Zhaoxin Liu and Junchang Kan. This is an open access article distributed under the Creative Commons Attribution License, which permits unrestricted use, distribution, and reproduction in any medium, provided the original work is properly cited.

\begin{abstract}
By accelerating the aforementioned industrialisation and urbanisation, people's way of life has undergone enormous changes. The incidence of overweight and obesity increases rapidly. This research first broke through the impact of basketball on improving physical health and then designed experiments separately. The experiment is divided into two experimental groups and a replacement group, with 150 people in total, 50 people per group, excluding the interference of gender, age, and other factors. The influencing factors are studied under the monitoring of the Internet of Things technology. One group had 8 weeks of basketball training, and the other group had 8 weeks of plant diet. The results showed that the average weight of the nutrition intervention group was reduced by $9.85 \mathrm{~kg}$, and the average weight of the exercise intervention group was reduced by $7.64 \mathrm{~kg}$. The percentage of body fat and blood lipid composition of the obese people have decreased, which indicates that the combination of basketball intervention under the monitoring of the Internet of Things technology can improve the physique of obese people.
\end{abstract}

\section{Introduction}

In recent years, with the change in the way people live, the rate of detection of overweight and obese people has been increasing every year. The following is the decline of physiological function and physical fitness of obese people, as well as the increase of metabolic diseases such as hypertension, diabetes, dyslipidemia, and metabolic syndrome. Keeping the body in a healthy weight range not only is related to their future study and life but also is a prerequisite for better service to the society in the future. Under the aesthetic standard of taking thinness as beauty, obesity obviously does not meet the aesthetic standards of contemporary people. What is more serious is that excessive obesity can also cause complications such as hypertension, hyperlipidemia, and type 2 diabetes. It is of great significance to strictly control the overweight and obesity rates and help obese college students reduce their obesity. Fat people not only have an impact on their social adaptation and psychology but also have a higher relative risk of obesity in adulthood than in childhood, which increases the frequency and mortality of chronic and cardiovascular diseases in adulthood. Therefore, it is very important to find effective and safe interventions to improve physical fitness and reduce the occurrence of metabolic diseases for obese adolescents.

Because of the importance of basketball intervention in obese people's physical health, many research teams began to study basketball and its mechanism and achieved good results. Gan, through 20 days of basketball training for 40 obese teenagers, found that basketball training can improve the body composition of young obese people. Traditional aerobic jogging can improve the body composition of obese teenagers, while basketball training can improve the body composition of obese teenagers with the same effect as jogging. Basketball training, as a way of fat reduction for obese teenagers, can reduce the negative emotional state and effectively improve the mood state of 
obese teenagers [1]. Zhou divided 60 students into experimental group and control group for 8 weeks. He found that taking part in basketball physical education can keep students' body composition in the normal range and achieve the effect of exercise and fat reduction. Participation in basketball sports can significantly improve the attractiveness of students, the vertical leap, and height (girls and boys), and muscle capacity can be improved effectively. Participation in physical basketball training has little impact on flexibility and the ability of high school students [2]. Taking part in basketball physical education can improve high school students' cardiopulmonary fitness, lung capacity, and maximal oxygen uptake. Although the research results are rich, there are still some deficiencies, mainly reflected in the limitations of the internal examination of obese people.

As people pay more and more attention to health problems, obesity has been paid more and more attention. Obesity is an abnormal accumulation of fat in the body. Adipose tissue secretes a variety of inflammatory factors and participates in the formation of metabolic syndrome such as insulin resistance. However, the cause of increased inflammatory factors in obese patients is unknown. Cao compared the fatty acid profile, dehydrogenase activity, and inflammatory factors' levels between obese and nonobese patients and analyzed the correlation between fatty acid profile, inflammatory factors, and insulin resistance index. It was found that the fatty acid profiles in plasma and adipose tissue were significantly different between the obese group and the nonobese group. The differences of fatty acid profile and inflammatory factors' levels in different parts of adipose tissue showed different metabolic activities, and this correlation was especially significant in obese patients [3]. Zhao randomly studied two classes and used the comprehensive intervention method of dietary nutrition health education and physical activity for one year and observed the intervention results (changes of obesity-related indicators and serum biochemical detection). It is found that the comprehensive intervention method of nutrition and health education for students, parents, teachers, and canteen staff and increasing students' physical activities can significantly reduce the incidence of childhood obesity. The intervention measures can regulate obesity-related indexes (waist circumference and waist height ratio) and blood biochemical indexes (cholesterol and low-density lipoprotein) [4]. Nowadays, the problem of obesity has been greatly improved, but it is not enough to study the effect of reducing fat scientifically and accurately without rebound. Therefore, it is the focus of current research to screen out the suitable health intervention for obese people.

The experiment uses a comparative method under the monitoring of the Internet of Things technology to study the impact of basketball and medicinal plants on the health of obese people. It mainly compares the population intervention of different factors of obese people under normal circumstances and observes the impact of basketball and medicinal plants on the health of obese people.

\section{Effect of Basketball and Medicinal Plants on Improving the Health of Obese People}

2.1. Influence of Basketball on Health. Basketball requires players to run and jump constantly on the field. Basketball will make human muscle fibers thicken, and muscle fiber diameter and cross section will increase. Strength training can change fast muscle fibers into slow muscle fiber and increase muscle volume. In the process of playing basketball, stretching muscles repeatedly can promote the increase of tendon and ligament cells, increase the muscle adventitia and intramuscular tension, make the muscle become strong, and improve the antitraction ability. Basketball is a sport that combines strength, speed, endurance, agility, and explosive power. The increase of speed makes the fast muscle fiber thicker, and the increase of endurance makes the number and volume of muscle fiber mitochondria increase. In addition, due to the flexible movement of each joint, the position, direction, and speed of the body have changed, thus improving the coordination of the whole muscle group, and the speed of muscle contraction is faster. Regular participation in basketball can increase muscle glycogen content, improve muscle oxygen storage capacity, reduce lactic acid production, delay the occurrence of sports fatigue, and improve muscle endurance. Basketball has an effect on body composition. Relevant data show that, in male college students, through long-term basketball training, upper arm sebum, part of sebum, and abdominal sebum thickness are significantly reduced. Basketball can make the body size such as chest circumference, waist circumference, and thigh circumference change significantly before and after sports. In addition, basketball is an aerobic exercise, which can increase the ugly activity of the fetus. With the increase of the amount of basketball exercise, the body fat decomposition speed is accelerated, the utilization rate of fat is improved, the lean weight is increased, and the body fat rate is reduced, which makes the human body more beautiful [5]. Basketball is characterized by long time and high intensity. During the exercise, the myocardial contractile force can be enhanced. Because of the large amount of exercise, it can make myocardial thickening, cardiac cavity expansion, and venous reflux increase. When the body is quiet, the lung ventilation is about 6 to 8 liters per minute. However, long-term basketball can increase the depth of breathing, reduce the respiratory rate, and increase the maximum oxygen uptake of human body [6]. The basketball game is divided into four quarters. The game time is about 45 minutes. The whole basketball game is played under the condition of fierce confrontation. The attacking team must complete an attack within 24 seconds, and the defenders should actively press, defend, attack, and grab. In the basketball court, quick and instantaneous actions such as jump shots, breakthroughs, and blocks need to rely on the energy of anaerobic metabolism, mainly the phosphate system and the glycolysis system; high-intensity sports such as continuous offensive and defensive conversion and full-court dribbling need to rely on the lactic acid function system to supplement energy; the athlete runs 180-200 times back and forth about 5400-6000 meters, which mainly depends on the oxidation 
energy supply system to provide energy. Therefore, the technical movements in basketball games are mainly provided by the original phosphate system, supplemented by lactic acid energy and aerobic oxidation system [7].

2.2. Influence of Body Mass Index on Disease. The relationship between body mass index and disease has always been the focus of attention. Low weight is an important factor leading to disease. Excessive weight loss will not alleviate the disease but will bring more problems. Of course, like low weight, excessive weight and obesity can cause problems. It is precisely because of the rapid increase in the rates of overweight and obesity which has caused widespread concern from all areas of life in the community, which has led to an increase in the study of the effects of obesity on health. Although studies on the impact of obesity on health emerge in endlessly and the research contents are different, the research results are basically the negative effects of obesity on health. Therefore, the World Health Organization has listed obesity as one of the five major health risk factors. In addition to causing a variety of diseases, the relationship between body mass index and mortality is also inseparable. There was an L-shaped relationship between BMI and total mortality. Underweight is a risk factor for increased overall mortality. Underweight is a prognostic factor in patients with gastric cancer. Multivariate analysis also showed that underweight increased mortality. Obesity is also considered an independent risk factor for cardiovascular disease and death. Epidemiological studies have shown that obesity is positively correlated with mortality (especially cardiovascular disease mortality), while BMI is negatively correlated with mortality. Compared with normal or low-weight people, overweight or obese people have better prognosis. In conclusion, although obesity is one of the main risk factors for many chronic diseases, the prognosis of obese patients may be better, so we should treat the impact of obesity on health dialectically. At the same time, the negative impact of underweight on mortality should not be ignored [8].

Body mass index (BMI) is an important index reflecting the relationship between body weight and height, which is closely related to body composition. In June 2002, the China Office of the International Society for Life Sciences held a seminar on "obesity and disease risk in China (WGOC)." The results show that Chinese adults with body mass index (BMI) less than 18.5 are underweight, those with BMI greater than or equal to 24.0 are overweight, and those with BMI greater than or equal to 28.0 are obese. In the actual research, because the people who are underweight are often far less than those with normal weight, overweight, and obesity, the research on the relationship between underweight and health is relatively rare, and most of the researches focus on overweight and obese people. The incidence rate of obesity is a chronic disease that causes weight to be over the normal. With the development of economy, the improvement of people's living standard, the change of lifestyle, and diet structure, overweight and obesity have gradually become an important factor in people's healthy life [9].
2.3. Effect of Medicinal Plant Intervention on Physical Fitness. At present, there are mainly three ways to reduce the weight of obese people by limiting energy intake: the first method is diet therapy, with energy intake of about $1200-1800 \mathrm{kcal}$ per day; the second method is low-energy therapy (LET), with energy intake of about $600-1000 \mathrm{kcal}$ per day; it is suitable for mild and moderate obesity; the third method is lowenergy treatment with low-energy intake every day, that is, about $1800 \mathrm{kcal}$ per day and less than $600 \mathrm{kcal}$ per day, which is suitable for severe and malignant obesity. These methods have different effects on different age groups of obese people, and the effect of teenagers is better than that of teenagers and middle-aged people. VLCDs at 3-4 months can reduce body weight by $15 \%-25 \%$, but the long-term effect is still controversial [10].

Currently, there are few studies on medicinal plants in China. The usual weight loss characteristics of medicinal plants include low fat, low calories, low blood sugar, and high fibers. Compared with other nutritional interventions, medicinal plants can better ensure adequate intake of multiple vitamins and minerals and can not only safely and effectively maintain weight loss but also improve weightrelated disease risk factors. The combination of medicinal plants and exercise has a good regulatory effect on the body composition, physiological function, physique, and fat and sugar metabolism of obese people. At present, the comprehensive evaluation of exercise and nutrition and the combination of exercise and medicinal plants are still very limited. Taking into account the individual differences in the constitution and metabolism of obese people, it is necessary to choose a suitable individualized program for them [11].

2.4. Health of Obese People. Obesity can cause metabolic syndrome such as hypertension, hyperlipidemia, and insulin resistance, lead to type 2 diabetes, cardiovascular disease, and cancer, and ultimately increase mortality. The prevention and treatment of obesity will produce great social and economic benefits. Obesity is an important risk factor of metabolic syndrome, and the levels of inflammatory factors in obese patients are increased. Obesity-related inflammation plays an important role in the occurrence and development of cancer. Therefore, early intervention of obesity can prevent the occurrence of a variety of diseases and reduce the overall mortality. Obesity is caused by environmental and genetic factors. Although genetic factors play an important role in the formation of obesity, environmental factors are still considered to be the main reason for the rapid growth of overweight and obesity in recent years. As an environmental factor, intestinal flora affects the health of host and participates in energy metabolism and disease formation of host. The role of intestinal flora in obesity has been gradually recognized [12]. In obese people, intestinal flora is more likely to absorb heat from food, and intestinal flora associated with obesity may improve the ability to absorb heat and subsequently cause related chronic inflammation. The change of intestinal flora improves intestinal permeability, makes endotoxin produced by Gramnegative bacteria enter the blood, and stimulates the body's 
chronic mild inflammation and the release of inflammatory factors, leading to and aggravating obesity and metabolic syndrome. Therefore, regulating intestinal flora through diet may be one of the strategies to treat or manage obesity in the future. The intervention of polyunsaturated fatty acids on obesity and metabolic syndrome is still controversial at home and abroad. The objective is to investigate the effects of polyunsaturated fatty acids (PUFA) on obesity and metabolic syndrome. Unhealthy eating habits are an important environmental factor leading to obesity and increased body fat. Excessive energy intake, especially fat intake, will lead to the imbalance of energy intake and consumption, increase the abnormal storage of fat, and lead to obesity and metabolic syndrome. The distribution of fatty acids in serum and tissues reflects the intake of fatty acids in diet and the synthesis of fatty acids in vivo. Analyzing the fatty acid profile and fat metabolism of obese patients and to study its role in metabolic syndrome and obesity-related inflammation will help to guide the diet of obese patients, reduce the risk of complications of obese patients, and promote health [13].

\section{The Impact of Basketball and Medicinal Plants on Improving the Health of Obese People under the Monitoring of IoT Technology}

3.1. Subjects. According to 50 obese people who participated in the exercise weight loss class in the academic year of 2018-2019, the obese people with $\mathrm{BMI} \geq 28$ were divided into exercise group (divided into male group and female group), and 50 obese people with $\mathrm{BMI} \geq 28$ were taken as nutrition group (divided into male group and female group), in addition to 50 obese people with $\mathrm{BMI} \geq 28$ as control group (divided into male group and female group). Three groups were studied. Among them, there were 78 boys, accounting for $52 \%$, and 72 girls, accounting for $48 \%$. All subjects signed the consent form before the experiment. Patients with endocrine system diseases, congenital heart diseases, and cardiovascular diseases were excluded by medical history investigation and physical examination before exercise. There was no significant difference in age, gender, and height among the three groups $(P>0.05)$.

3.2. Experimental Design. According to the results of physical fitness test, the subjects were divided into two groups, respectively, given nutritional food and basketball intervention. The physical state test was carried out once a week to study the effects of exercise and nutritional intervention on the physical state of the test animals through selfcontrol. The whole experiment is divided into three steps. The first step is to test the health and physical fitness of the subjects before the experiment. In order to ensure that there is no significant difference between the two groups in the test indicators of health and physical fitness, we need to fine-tune a single sample for a week; the second step is the experimental stage, a total of eight weeks, to ensure that the experimental group and the control group have the same teaching time, teaching environment, and other factors; the teaching time is three sessions a week, with the duration of each session being 40 minutes. In the experiment, the control group was taught according to the teaching content and organization form of "physical education and health curriculum standard." The experimental group carried out basketball physical education. The third step is the experimental measurement after the end of the experiment; the period is one week. 1-2 lectures on nutrition knowledge are organized to teach the experimental subjects the methods of reasonable diet. According to the dietary characteristics of different groups, a simple popular science page was prepared or sent to the experimental subjects according to the diet template. Regularly supervise and spot check the dining situation of the experimental subjects is regularly supervised and spot-checked, and the existing problems are pointed out.

3.3. Mathematical Statistics under the Monitoring of the Internet of Things Technology. Based on the principle of sports statistics, this study used SPSS 17.0 statistical software to analyze the differences of basketball and nutrition intervention on the test indexes of healthy physical fitness. The differences of the physical fitness data between the two groups of subjects and the control group before and after the experiment were tested. The objective and effective data provide more powerful evidence for this paper.

\section{Discussions}

4.1. Changes of Body Weight Index after Experimental Intervention. 150 obese college students were given exercise intervention and nutrition intervention for 8 weeks. The subjects were tested once a week. The main indexes included body weight, BMI, and body fat rate. The following table is the average body weight change table of the subjects in 8 weeks; from left to right, we have women in the exercise intervention group, men in the exercise intervention group, women in the nutrition group, and men in the nutrition group. The average weight of female nutrition group decreased from $89.61 \mathrm{~kg}$ to $81.0 \mathrm{~kg}$, a total of $8.61 \mathrm{~kg}$; the average weight of male nutrition group decreased from $101.53 \mathrm{~kg}$ to $90.68 \mathrm{~kg}$, a total of $10.85 \mathrm{~kg}$. The average weight of women in the exercise intervention group decreased from $92.15 \mathrm{~kg}$ to $85.41 \mathrm{~kg}$, a total of $6.74 \mathrm{~kg}$. The average weight of males in the exercise intervention group decreased from $99.32 \mathrm{~kg}$ to $92.90 \mathrm{~kg}$, a total of $6.42 \mathrm{~kg}$, while there was no significant change in the control group. See Table 1 for details.

The figure above shows the average weight change of the subjects within 8 weeks. This chart can clearly reflect the weight change of subjects within 8 weeks. In the following picture, the blue line is the male group, the orange line is the female group, the grey line is the male food group, and the yellow line is the female food group. As shown in the figure below, the weight loss effect of nutritional male group is better than that of exercise intervention male group. In the male group, the body weight decreased by $0.4 \mathrm{~kg}$ in the first 
TABLE 1: Weight changes of subjects in eight weeks.

\begin{tabular}{|c|c|c|c|c|}
\hline & $\begin{array}{l}\text { Exercise intervention } \\
\text { group (female) }\end{array}$ & $\begin{array}{l}\text { Exercise intervention } \\
\text { group (male) }\end{array}$ & $\begin{array}{c}\text { Nutrition } \\
\text { intervention } \\
\text { group (female) }\end{array}$ & $\begin{array}{c}\text { Nutrition } \\
\text { intervention } \\
\text { group (male) }\end{array}$ \\
\hline Initial value & 92.1 & 99.3 & 89.6 & 101.5 \\
\hline First week & 91.8 & 98.9 & 88.8 & 101.1 \\
\hline Second week & 91.1 & 99.1 & 87.8 & 99.3 \\
\hline Third week & 91.3 & 97.9 & 86.5 & 97.9 \\
\hline Fourth week & 89.5 & 96.1 & 85.1 & 97.1 \\
\hline Fifth week & 88.3 & 95.2 & 84.3 & 95.5 \\
\hline Sixth week & 87.8 & 94.8 & 82.9 & 94.2 \\
\hline Seventh week & 86.3 & 93.8 & 81.8 & 92.8 \\
\hline Eighth week & 85.4 & 92.9 & 81.0 & 90.6 \\
\hline
\end{tabular}

week, increased by $0.2 \mathrm{~kg}$ in the second week, increased by $1.2 \mathrm{~kg}$ in the third week, increased by $1.8 \mathrm{~kg}$ in the fourth week, increased by $0.9 \mathrm{~kg}$ in the fifth week, increased by $0.4 \mathrm{~kg}$ in the sixth week, increased by $1.0 \mathrm{~kg}$ in the seventh week, and increased by $0.9 \mathrm{~kg}$ in the eighth week. In the nutritional male group, the body weight decreased by $0.4 \mathrm{~kg}$ in the first week, $1.8 \mathrm{~kg}$ in the second week, $1.4 \mathrm{~kg}$ in the third week, $0.8 \mathrm{~kg}$ in the fourth week, $1.6 \mathrm{~kg}$ in the fifth week, $1.3 \mathrm{~kg}$ in the sixth week, $1.4 \mathrm{~kg}$ in the seventh week, and $2.2 \mathrm{~kg}$ in the eighth week. The body weight of nutrition male group showed a downward trend, and the decline rate was gentle. The male exercise intervention group showed a downward trend in the 1st and 3rd weeks, but the change range was not obvious, and the weight decreased rapidly from the 5 th week to the 8 th week.

From the point of view of weight loss effect, the effect of nutrition intervention group was significantly better than that of exercise intervention group. In the male nutrition group, it decreased at a constant rate every week. In the first two weeks and the third week, the speed of weight loss was slower in the male group of exercise intervention, but it showed an upward trend in the second week. The fifth week showed a significant downward trend. In two groups of subjects exercise intervention program was consistent, but weekly weight loss effect was not consistent; analysis of the reasons is as follows: exercise intervention male group did not carry out reasonable diet control, resulting in slow weight loss. From the fifth week to the eighth week, the body weight dropped rapidly. Under the pressure of exercise performance, male subjects in the exercise intervention group adopted diet to promote rapid weight loss. The nutrition group had a good diet control during the experimental period, hence the average weight loss during the experimental intervention. The details are shown in Figure 1.

As shown in Figure 2, the yellow line represents the nutritional women group, and the orange line represents the exercise intervention group. As regards the extent of the loss of weight, the physical weight of the intervention group was reduced by $8.6 \mathrm{~kg}$ in eight weeks, and the weight of the intervention group was reduced by $7.94 \mathrm{~kg}$ in eight weeks. The weight loss effect of nutrition intervention group was better than that of exercise intervention group. From the point of view of weekly weight loss effect, the body weight of nutritional women group decreased by $0.8 \mathrm{~kg}$ in the first

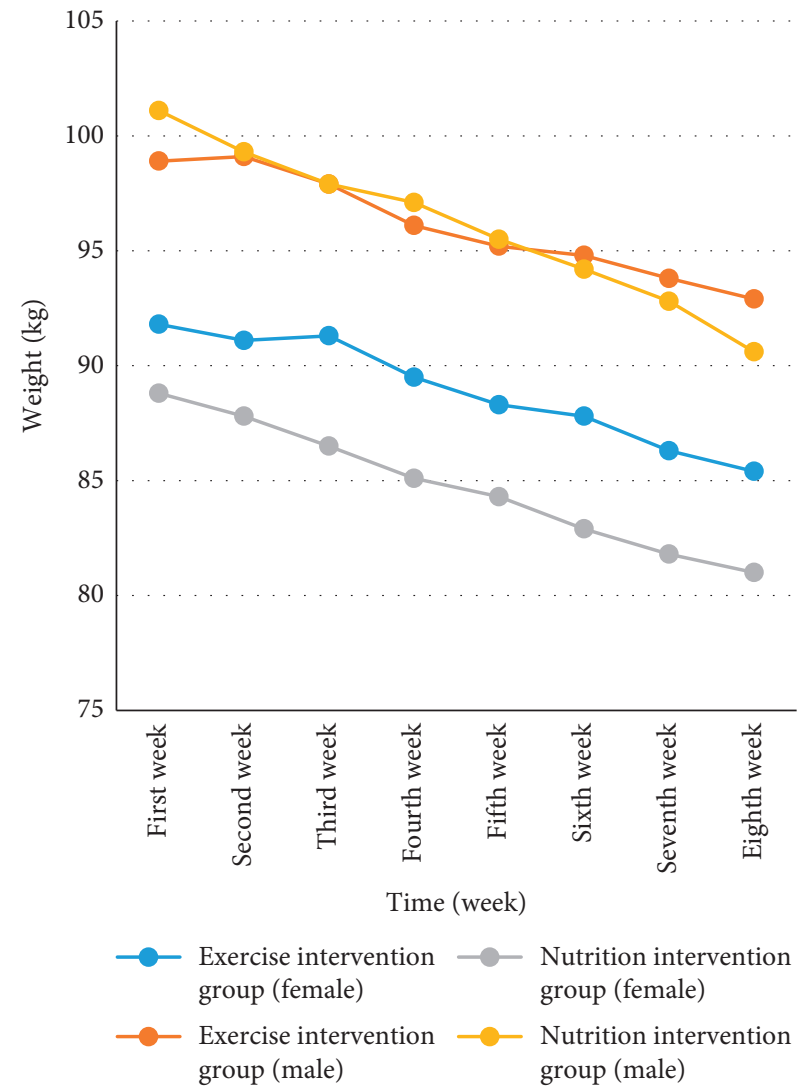

FIGURE 1: Weight changes of each experimental group during the experiment.

week, $1.0 \mathrm{~kg}$ in the second week, $1.3 \mathrm{~kg}$ in the third week, $1.4 \mathrm{~kg}$ in the fourth week, $0.8 \mathrm{~kg}$ in the fifth week, $1.4 \mathrm{~kg}$ in the sixth week, $1.1 \mathrm{~kg}$ in the seventh week, and $0.8 \mathrm{~kg}$ in the eighth week. In the nutrition group, the body weight decreased evenly. In the exercise intervention group, the body weight decreased by $0.3 \mathrm{~kg}$ in the first week, $0.7 \mathrm{~kg}$ in the second week, $0.2 \mathrm{~kg}$ in the third week, $1.8 \mathrm{~kg}$ in the fourth week, $1.2 \mathrm{~g}$ in the fifth week, $0.5 \mathrm{~kg}$ in the sixth week, $1.5 \mathrm{~kg}$ in the seventh week, and $0.9 \mathrm{~kg}$ in the eighth week. From the weight loss effect, the weight of the nutrition group showed a downward trend every week, while the weight of the exercise intervention group showed a downward trend in the first 


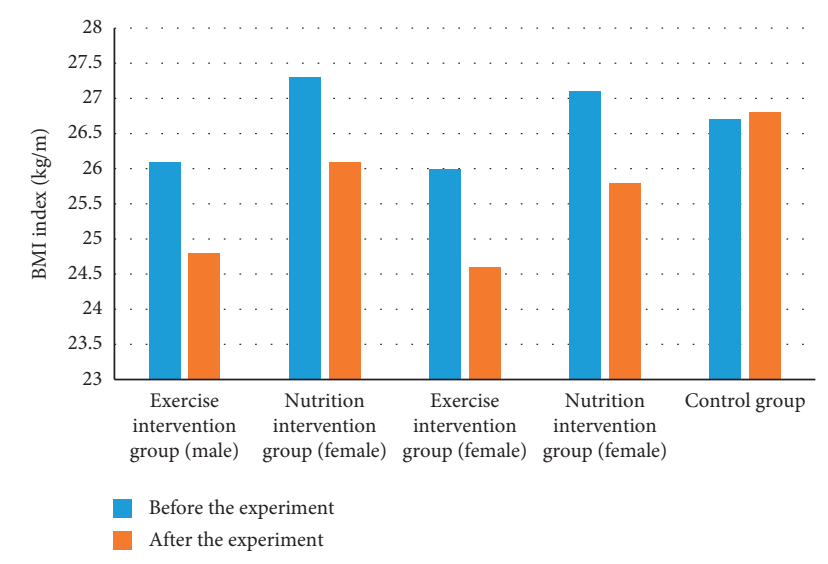

FIGURE 2: The changes of BMI before and after the experiment.

and second weeks, but the decline rate was relatively slow. At the third week, the weight of female group showed an upward trend, and the weight of female group decreased from the fifth week to the eighth week. The analysis of the specific reasons is as follows: exercise intervention women did not carry out reasonable diet control four weeks before exercise intervention, so they lost weight slowly. From the 5 th week to the 8 th week, the subjects were over dieting during the exercise intervention under the pressure of the end of the experiment. It can be seen from the above that the body weight of the exercise intervention group decreased slowly in the first and second weeks and even increased in the third week, while the weight decreased rapidly from the fifth week to the eighth week. However, during the experimental intervention, the body weight of the nutrition group showed a uniform downward trend. From the weekly weight loss effect and total weight loss effect, the nutrition group was better than the exercise intervention group.

\subsection{Change Analysis of Body Composition in Each Group.} Through the implementation of different intervention programs for eight weeks, the body weight of the exercise group and the nutrition group in the experimental group decreased, while the weight of the nutrition group decreased significantly. Both groups were significantly lower. After the experiment, the fat weight of exercise group and nutrition group decreased significantly. At the same time, the lean weight of both experimental groups increased, and the increase of exercise group was very significant. The decrease of BMI in both experimental groups was very significant $(P<0.01)$, as shown in Figures 2 and 3.

After the experiment, the BMI of exercise group and nutrition group decreased, respectively, and the decrease of two groups was very significant. At the same time, the control group was more than before. As shown in Figure 3, eight weeks of exercise and nutrition intervention can reduce the BMI of overweight and obese people. The effect of nutrition group was better than that of the two schemes.

After eight weeks of intervention, the fat weight of exercise group and nutrition group decreased by $1.4 \mathrm{~kg}$ and $1.1 \mathrm{~kg}$, respectively, and the decrease in both groups was very

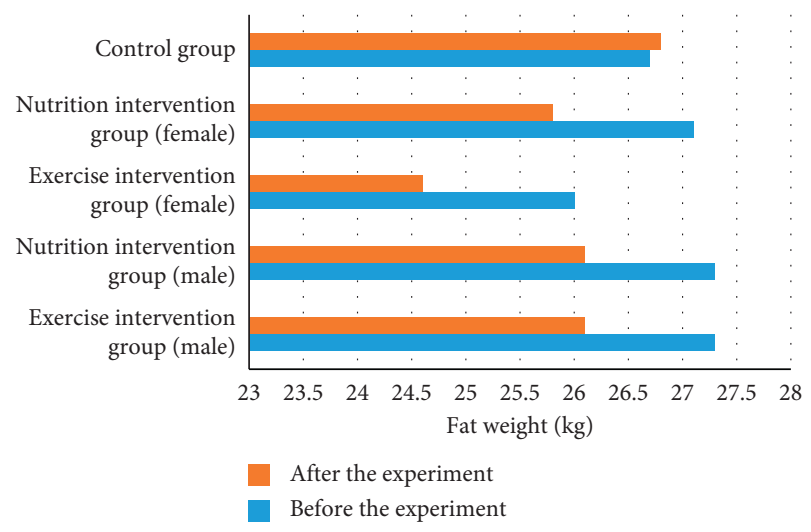

Figure 3: Changes of fat weight before and after the experiment.

significant. It can be seen that eight weeks of exercise and nutrition intervention can reduce the fat weight of obese adolescents, and nutrition intervention has the best effect on reducing fat weight of overweight and obese people. Therefore, eight weeks of basketball can increase the lean weight of obese people. Although some studies have shown that energy restriction may reduce lean body weight, the results of this study show that the total energy provided to overweight and obese people and normal weight, respectively, does not affect the development of lean body weight. Studies have shown that reducing the weight of obese girls can stimulate the release of growth hormone. But, on the whole, weekly aerobic exercise, nutrition intervention, and exercise nutrition intervention can reduce the body fat percentage of overweight and obese adolescents. Therefore, by comprehensive comparison of the effects of weekly aerobic exercise, nutritional intervention, and exercise nutrition intervention programs on the body fat percentage of overweight and obese adolescents in this study, the three intervention programs can effectively reduce the obesity degree of overweight and obese adolescents.

4.3. Changes of Blood Lipids. Through the implementation of the eight-week intervention program, the TC of exercise group and nutrition group were significantly decreased $(P<0.05$ and $P<0.01)$. The LDL-C of the two experimental groups also decreased, especially in the nutrition group. The HDL-C in the experimental group and the control group were significantly decreased $(P<0.01)$. There was no significant change in TG before and after the experiment, as shown in Figure 4.

There are many items to detect blood lipids in clinic. The basic detection items of blood lipids are TC, TG, HDL-C, and LDL-C, which are usually called "four blood lipids." According to the Chinese guidelines for dyslipidemia in adults, the four tests are the sum of cholesterol in each lipoprotein in the blood. The results of a cohort study in China showed that, with the increase of TC, the risk of ischemic cardiovascular disease also increased, and there was no turning point in the relationship between TC and risk. When TC was between $5.18 \mathrm{mmol} / L$ and $6.19 \mathrm{mmol} / L$, the risk of ischemic cardiovascular disease increased. When $\mathrm{TC} \geq 6.22 \mathrm{mmol} / L$, the risk was more than 2 times of 


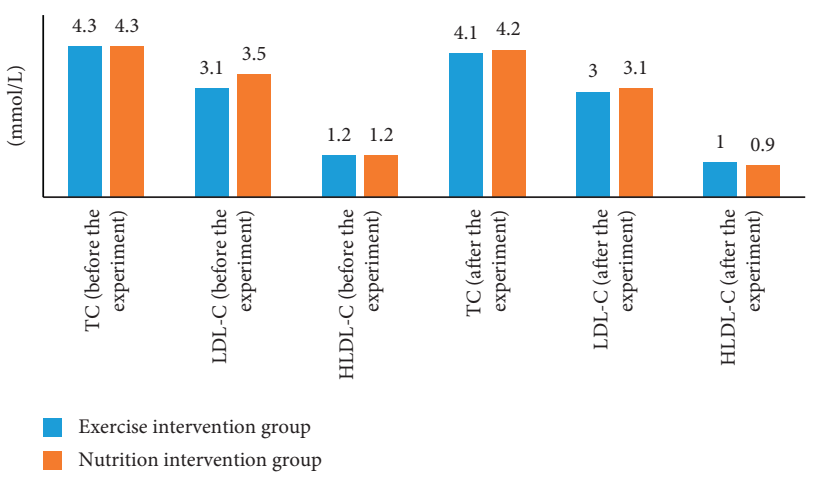

Figure 4: Changes of TC, LDL-C, and HDL-C before and after the experiment.

$3.63 \mathrm{mmol} / L$, and the appropriate value of TC should be less than $5.18 \mathrm{mmol} / \mathrm{L}$. The decrease of TC in exercise group and nutrition group was significantly lower than that in control group. It can be seen that the two intervention programs of 8 weeks can reduce the TC of obese people, indicating that these three programs can reduce the risk of ischemic cardiovascular disease in obese people.

LDL-C is the basic factor of atherosclerosis. As an indicator of total blood volume, LDL-C is similar to the incidence trend of cardiovascular disease and the degree of TC. In this study, after the experiment, LDL-C of exercise group and nutrition group decreased, and only the nutrition group had significant difference. The reason why there is no significant difference in exercise group may be related to lowdensity lipoprotein cholesterol before the experiment. In addition, the continuous changes of LDL-C and ischemic cardiovascular disease indicate that the decrease of LDL-C can reduce the occurrence of atherosclerosis.

HDL-C is generally considered as an atherosclerotic lipoprotein in vivo. There is no direct and comprehensive method to detect the content and function of HDL-C. Therefore, we can know the content of HDL-C in plasma indirectly by detecting the content of cholesterol. Studies have shown that the decrease of HDL-C is related to the occurrence of ischemic diseases. When HDL-C $<1.04 \mathrm{mmol} / L$, the risk of ischemic disease increased by $50 \%$. The results showed that, after 8 weeks, all experimental groups, including the control group, showed a downward trend. This may be due to the fact that the average values of the three groups are still in the appropriate range, and the occurrence of HDL-C and cardiovascular disease does not show the continuity similar to TC and LDL-C, and there is no turning point phenomenon. Therefore, the effect of exercise and nutrition on overweight and obese people with normal HDLC needs further study.

\section{Conclusion}

After eight weeks of basketball training, under the supervision of the Internet of Things technology, the following changes in indicators, weight, weight, BMI, body aunt, body sulphur, body water, muscle, mineral elements, and other objects, are significantly homogeneous, resulting in a very significant difference. Basketball training can improve the physical fitness of obese people. The intervention group received eight weeks of basketball training.

In this study, the cardiopulmonary function and physical fitness of overweight and obese people are worse than those of normal people: eight weeks of exercise and nutrition can effectively alleviate the obesity of overweight and obese people. Exercise intervention can improve the cardiopulmonary function of overweight and obese people. The combination of basketball and medicinal plant intervention has the best effect on reducing visceral feces and improving physical fitness.

\section{Data Availability}

The data that support the findings of this study are available from the corresponding author upon reasonable request.

\section{Conflicts of Interest}

The authors declare no potential conflicts of interest with respect to the research, authorship, and/or publication of this article.

\section{References}

[1] S. P. Hagenaars, C. R. Gale, I. J. Deary, and S. E. Harris, "Cognitive ability and physical health: a mendelian randomization study," Scientific Reports, vol. 7, no. 1, p. 2651, 2017.

[2] K. Shifren and K. Anzaldi, "Optimism, well-being, depressive symptoms, and perceived physical health: a study among stroke survivors," Psychology Health \& Medicine, vol. 23, no. 1, p. 1, 2017.

[3] B. Matthew and L. Kangjae, "Within what distance does "greenness" best predict physical health? a systematic review of articles with gis buffer analyses across the lifespan," International Journal of Environmental Research and Public Health, vol. 14, no. 7, p. 675, 2017.

[4] G. J. P. D. Bernardin, L. Giancarlo, M. P. Rossi, V. Homero, and V. Gianni, "Complementary religious and spiritual interventions in physical health and quality of life: a systematic review of randomized controlled clinical trials," PLoS One, vol. 12, no. 10, Article ID e0186539, 2017.

[5] J. M. Brooks, E. Umucu, J. Sánchez, C. Seehusen, and S. J. Bartels, "Doctor-diagnosed arthritis and self-reported physical health function among middle-aged and older adults with serious mental illness," Journal of Nervous \& Mental Disease, vol. 207, no. 11, p. 1, 2019.

[6] M. Shi, M. Narayanasamy, C. Yang et al., "3D interpenetrating assembly of partially oxidized MXene confined Mn-Fe bimetallic oxide for superior energy storage in ionic liquid," Electrochimica Acta, vol. 334, Article ID 135546, 2020.

[7] L. M. Shank, N. A. Schvey, K. Ekundayo et al., "The relationship between weight stigma, weight bias internalization, and physical health in military personnel with or at high-risk of overweight/obesity," Body Image, vol. 28, pp. 25-33, 2019.

[8] M.-C. Lin, P.-W. Shueng, W.-K. Chang et al., "Consensus and clinical recommendations for nutritional intervention for head and neck cancer patients undergoing chemoradiotherapy in taiwan," Oral Oncology, vol. 81, pp. 16-21, 2018. 
[9] O. Katz-Shufan, T. Simon-Tuval, L. Sabag, and D. R. Shahar, "Improvement in healthy meal index, lunch quality, and diversity scores following an integrated nutritional intervention in a communal dining room: the nekst study," $\mathrm{Nu}$ trients, vol. 12, no. 6, p. 1741, 2020.

[10] A. M. Cuevas, M. Lazo, I. Zuñiga et al., "Expression of myd88 in adipose tissue of obese people: is there some role in the development of metabolic syndrome?" Metabolic Syndrome and Related Disorders, vol. 15, no. 2, pp. 80-85, 2017.

[11] Y. Poria, J. Beal, and A. Shani, "Does size matter? an exploratory study of the public dining experience of obese people," Journal of Hospitality and Tourism Management, vol. 39, pp. 49-56, 2019.

[12] S.-M. Hong, G.-H. Lee, and Y.-A. Shin, "Exercise strategies for prevention of musculoskeletal disorders of obese people," Journal of the Korean Society for Wellness, vol. 12, no. 4, pp. 655-663, 2017.

[13] S. Moon, C. M. Oh, M. K. Choi et al., "The influence of physical activity on risk of cardiovascular disease in people who are obese but metabolically healthy," Plos One, vol. 12, no. 9, Article ID e0185127, 2017. 